\title{
Neighbor-Locating Colorings in Graphs
}

\author{
Liliana Alcon*2, Marisa Gutierrez ${ }^{\dagger 2}$, Carmen Hernando ${ }^{\ddagger 1}$, Mercè Mora ${ }^{\S 1}$, and Ignacio \\ M. Pelayo $\$ 1$ \\ ${ }^{1}$ Departament de Matemàtiques, Universitat Politècnica de Catalunya \\ ${ }^{2}$ Centro de Matemáticas, Universidad Nacional de La Plata
}

\begin{abstract}
A $k$-coloring of a graph $G$ is a $k$-partition $\Pi=\left\{S_{1}, \ldots, S_{k}\right\}$ of $V(G)$ into independent sets, called colors. A $k$-coloring is called neighbor-locating if for every pair of vertices $u, v$ belonging to the same color $S_{i}$, the set of colors of the neighborhood of $u$ is different from the set of colors of the neighborhood of $v$. The neighbor-locating chromatic number $\chi_{N L}(G)$ is the minimum cardinality of a neighbor-locating coloring of $G$.

We establish some tight bounds for the neighbor-locating chromatic number of a graph, in terms of its order, maximum degree and independence number. We determine all connected graphs of order $n \geq 5$ with neighbor-locating chromatic number $n$ or $n-1$. We examine the neighbor-locating chromatic number for two graph operations: join and disjoint union, and also for two graph families: split graphs and Mycielski graphs.
\end{abstract}

Key words: coloring; domination; location; vertex partition; neighbor-locating coloring.

\section{Introduction}

Domination and location in graphs are two important subjects that have received much attention, usually separately, but sometimes also both together. There are mainly two types of location, metric location and neighbor location. In this work, we are interested in neighbor location, and we explore this concept in the particular context of a special kind of vertex partitions, called colorings.

Metric location in sets was simultaneously introduced by P. Slater [38] and F. Harary and R. A. Melter [23] and further studied in different contexts (see [7, 26]). In [25], M. A.

\footnotetext{
${ }^{*}$ Partially supported by PIP 11220150100703CO CONICET, liliana@mate.unlp.edu.ar

${ }^{\dagger}$ Partially supported by PIP 11220150100703 CO CONICET, marisa@mate.unlp.edu.ar

${ }^{\ddagger}$ Partially supported by projects MTM2015-63791-R (MINECO/FEDER) and Gen. Cat. DGR 2017SGR1336, carmen.hernando@upc.edu

${ }^{\S}$ Partially supported by projects MTM2015-63791-R (MINECO/FEDER), Gen. Cat. DGR 2017SGR1336 and H2020-MSCA-RISE project 734922-CONNECT, merce.mora@upc.edu

`Partially supported by projects MINECO MTM2014-60127-P, ignacio.m.pelayo@upc.edu
} 
Henning and O. R. Oellermann introduced the so-called metric-locating-dominating sets, by merging the concepts of metric-locating set and dominating set.

In [14], G. Chartrand, E. Salehi and P. Zhang, brought the notion of metric location to the ambit of vertex partitions, introducing the resolving partitions, also called metriclocating partition, and defining the partition dimension. Metric location and domination, in the context of vertex partitions, are studied in [28]. In [11], there were introduced the so-called locating colorings considering resolving partitions formed by independents sets.

Neighbor location in sets was introduced by P. Slater in [39]. Given a graph $G$, a set $S \subseteq V(G)$ is a dominating set if every vertex not in $S$ is adjacent to some vertex in $S$. A set $S \subseteq V(G)$ is a locating-dominating set if $S$ is a dominating set and $N(u) \cap S \neq N(v) \cap S$ for every two different vertices $u$ and $v$ not in $S$. The location-domination number of $G$, denoted by $\lambda(G)$, is the minimum cardinality of a locating-dominating set. In $[8,27]$, bounds for this parameter are given. In this paper, merging the concepts studied in [11, 39], we introduce the neighbor-locating colorings and the neighbor-locating chromatic number, and examine this parameter in some families of graphs.

The paper is organized as follows. In Section 2, we define the neighbor-locating colorings and introduce the neighbor-locating chromatic number of a graph. In Section 3, bounds for the neighbor-locating chromatic number of a graph are established in terms of its order, maximum degree and independence number. In Section 4, we focus our attention on graphs with neighbor-locating chromatic number close to the order $n$. Concretely, we characterize all graphs with neighbor-locating chromatic number equal to $n$ or to $n-1$. Section 5 is devoted to examining the neighbor-locating chromatic number for some graph operations: the join and the disjoint union. Section 6 is devoted to studying the neighbor-locating chromatic number of connected split graphs and Mycielski graphs. Finally, in Section 7, we pose several open problems.

We introduce now some basic terminology. All the graphs considered are undirected, simple and finite. The vertex set and the edge set of a graph $G$ are denoted by $V(G)$ and $E(G)$, respectively. If $u v \in E(G)$, then we write $u \sim v$. Let $v$ be a vertex of $G$. The open neighborhood of $v$ is $N_{G}(v)=\{w \in V(G): v w \in E(G)\}$, and the closed neighborhood of $v$ is $N_{G}[v]=N(v) \cup\{v\}$. The degree of $v$ is $\operatorname{deg}_{G}(v)=\left|N_{G}(v)\right|$. If $N_{G}[v]=V(G)$, then $v$ is called universal. If $\operatorname{deg}_{G}(v)=1$, then $v$ is called a leaf. An isolated vertex is a vertex of degree zero. Let $W$ be a subset of vertices of a graph $G$. The open neighborhood of $W$ is $N_{G}(W)=\cup_{v \in W} N_{G}(v)$, and the closed neighborhood of $W$ is $N_{G}[W]=N_{G}(W) \cup W$. The subgraph of $G$ induced by $W$, denoted by $G[W]$, has $W$ as vertex set and $E(G[W])=\{v w \in$ $E(G): v \in W, w \in W\}$. If a graph $H$ is an induced subgraph of $G$, then we write $H \prec G$.

The distance between vertices $v, w \in V(G)$ is denoted by $d_{G}(v, w)$, or $d(v, w)$ if the graph $G$ is clear from the context. The diameter of $G$ is $\operatorname{diam}(G)=\max \{d(v, w): v, w \in$ $V(G)\}$. The independence number of $G$, denoted by $\alpha(G)$, is the maximum cardinality of an independent set of $G$. For undefined terminology, we refer the reader to [13]. 


\section{Locating partitions}

In this section, we present different kinds of locating partitions that have been extensively studied in recent years and that are related to the partitions that we introduce in this paper: the neighbor-locating colorings.

Given a connected graph $G$, a vertex $v \in V(G)$ and a set of vertices $S \subseteq V(G)$, the distance $d(v, S)$ between $v$ and $S$ is $d(v, S)=\min \{d(v, w): w \in S\}$. Given a partition $\Pi=$ $\left\{S_{1}, \ldots, S_{k}\right\}$ of $V(G)$, we denote by $r(v \mid \Pi)$ the vector of distances between a vertex $v \in V(G)$ and the elements of $\Pi$, that is, $r(v \mid \Pi)=\left(d\left(v, S_{1}\right), \ldots, d\left(v, S_{k}\right)\right)$. The partition $\Pi$ is called a metric-locating partition, an $M L$-partition for short, if, for any pair of distinct vertices $u, v \in$ $V(G), r(u \mid \Pi) \neq r(v \mid \Pi)$. The partition dimension $\beta_{p}(G)$ of $G$ is the minimum cardinality of an ML-partition of $G$. Metric-locating partitions were introduced in [14], and further studied in several papers: bounds [10], graph families [16, 17, 20, 21, 24, 29, 30, 31, 36, 37, 41, 42] and graph operations $[2,9,15,18,19,35,46,47]$.

A partition $\Pi=\left\{S_{1}, \ldots, S_{k}\right\}$ of $V(G)$ is dominating if, for every $i \in\{1, \ldots, k\}$ and for every vertex $v \in S_{i}, d\left(v, S_{j}\right)=1$, for some $j \in\{1, \ldots, k\}$. The partition $\Pi$ is called a metric-locating-dominating partition, an $M L D$-partition for short, if it is both dominating and metric-locating. The partition metric-location-domination number $\eta_{p}(G)$ of $G$ is the minimum cardinality of an MLD-partition of $G$. In [28], it was proved that $\beta_{p}(G) \leq \eta_{p}(G) \leq \beta_{p}(G)+1$.

Let $\Pi=\left\{S_{1}, \ldots, S_{k}\right\}$ be a partition of $V(G)$. If all the parts of $\Pi$ are independent sets, then we say that $\Pi$ is a coloring of $G$ and that the elements of $S_{i}$ are colored with color $i$. The chromatic number $\chi(G)$ equals the minimum cardinality of a coloring of $G$.

A coloring $\Pi=\left\{S_{1}, \ldots, S_{k}\right\}$ is called a (metric-)locating coloring, an ML-coloring for short, if for every $i \in\{1, \ldots, k\}$ and for every pair of distinct vertices $u, v \in S_{i}$, there exists $j \in\{1, \ldots, k\}$ such that $d\left(u, S_{j}\right) \neq d\left(v, S_{j}\right)$. In other words, an ML-coloring $\Pi$ is a coloring that is also an ML-partition. The (metric-)locating-chromatic number $\chi_{L}(G)$ is the minimum cardinality of an ML-coloring of $G$. This parameter was introduced in [11] and further studied in $[4,5,6,12,24,33,34,40,43,44,45]$.

In this paper, we introduce a new type of locating coloring. If in the previous paragraph the location was in terms of distances (and just for connected graphs), now we focus our attention on the neighbors.

Definition 1. Let $G$ be a graph $G$, not necessarily connected. A coloring $\Pi=\left\{S_{1}, \ldots, S_{k}\right\}$ is called a neighbor-locating coloring, an $N L$-coloring for short, if for every pair of different vertices $u, v$ belonging to the same color $S_{i}$, the set of colors of the neighborhood of $u$ is different from the set of colors of the neighborhood of $v$, that is, $\left\{j: 1 \leq j \leq k, N(u) \cap S_{j} \neq\right.$ $\emptyset\} \neq\left\{j: 1 \leq j \leq k, N(v) \cap S_{j} \neq \emptyset\right\}$.

The neighbor-locating chromatic number $\chi_{N L}(G)$, the $N L C$-number for short, is the minimum cardinality of an NL-coloring of $G$.

Remark 1. Let $\Pi$ be an NL-coloring of a graph $G$. If $G$ is a non-connected graph with isolated vertices, then $\Pi$ is not a dominating partition. Conversely, if $G$ is either a connected graph or a non-connected graph without isolated vertices, then $\Pi$ is also a dominating partition.

Remark 2. A coloring $\Pi=\left\{S_{1}, \ldots, S_{k}\right\}$ is an NL-coloring if, for every $i \in\{1, \ldots, k\}$ and for every pair of distinct non-isolated vertices $u, v \in S_{i}$, there exists $j \in\{1, \ldots, k\}$ such that 
either $d\left(u, S_{j}\right)=1$ and $d\left(v, S_{j}\right) \neq 1$ or $d\left(u, S_{j}\right) \neq 1$ and $d\left(v, S_{j}\right)=1$ and there is at most one isolated vertex of color $i$, for every $i \in\{1, \ldots, k\}$.

Remark 3. Let $\Pi=\left\{S_{1}, \ldots, S_{k}\right\}$ be a partition of the set $V(G)$ of vertices of a graph $G$. For every vertex $x \in V(G)$, we define the tuple $n r(x \mid \Pi)=\left(x_{1}, \ldots, x_{k}\right)$ as follows

$$
x_{i}= \begin{cases}0, & \text { if } x \in S_{i} ; \\ 1, & \text { if } x \in N\left(S_{i}\right) \backslash S_{i} \\ 2, & \text { if } x \notin N\left[S_{i}\right] .\end{cases}
$$

Observe that, if $G$ is connected, then $x_{i}=\min \left\{2, d\left(x, S_{i}\right)\right\}$. Notice that $n r(x \mid \Pi)$ has exactly one component equal to 0 . In fact, $S_{i}$ contains exactly all the vertices $x \in V(G)$ such that the i-th component of $n r(x \mid \Pi)$ is equal to 0 . With this terminology, $\Pi$ is an NL-coloring if and only if the sets $S_{1}, \ldots, S_{k}$ are independent and $n r(x \mid \Pi) \neq n r(y \mid \Pi)$, for every pair of distinct vertices $x$ and $y$. Moreover, if $\Pi$ is an NL-coloring of $G$ and $x \in V(G)$ is a non isolated vertex, then the tuple $n r(x \mid \Pi)$ has at least one component equal to 1.

Given a graph $G$, an NL-coloring $\Pi$ of $V(G)$ and two vertices $x, y \in V(G)$, if $n r(x \mid \Pi) \neq$ $n r(y \mid \Pi)$, then $x, y$ are said to be neighbor-located by $\Pi$.

Remark 4. If $G$ is a graph and $W \subseteq V(G)$ is the set of all isolated vertices of $G$, then

$$
\chi_{N L}(G)=\max \left\{\chi_{N L}(G[V(G) \backslash W]),|W|\right\} .
$$

The following chains of inequalities hold.

Proposition 1. Let $G$ be a non-trivial graph. Then,

(1) $2 \leq \chi(G) \leq \chi_{L}(G) \leq \chi_{N L}(G)$.

(2) $2 \leq \beta_{p}(G) \leq \eta_{p}(G) \leq \chi_{N L}(G)$.

(3) $\chi_{N L}(G) \leq \chi(G)+\lambda(G)$.

Proof. Items (1) and (2) are a direct consequence of the definitions. To prove (3), take a minimum locating-dominating set $W$ of $G$. Let $\mathrm{H}$ be the subgraph of $G$ induced by $V(G) \backslash W$. Take a $k$-coloring $\Pi_{H}=\left\{S_{1}, \ldots, S_{k}\right\}$ of $H$, where $k=\chi(H)$. Then, $\Pi=\Pi_{H} \cup\{\{w\}: w \in W\}$ is an NL-coloring of $G$. As $\chi(H) \leq \chi(G)$, we obtain that the inequality is satisfied.

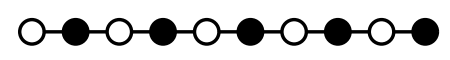

(a)

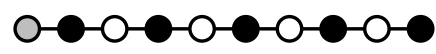

(b)

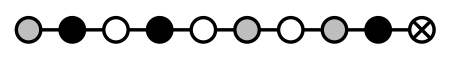

(c)

Figure 1: (a) $\chi\left(P_{10}\right)=2$; (b) $\chi_{L}\left(P_{10}\right)=3$ and (c) $\chi_{N L}\left(P_{10}\right)=4$.

Proposition 2. For each pair $h, k$ of integers with $3 \leq h \leq k$, there exists a connected graph $G$ with $\chi(G)=h$ and $\chi_{N L}(G)=k$.

Proof. It is enough to consider the graph obtained from the complete graph $K_{h}$ by hanging $k-1$ leaves to one of its vertices. 


\section{Bounds}

This section is devoted to establishing some bounds involving the NLC-number, the order, the diameter and the independence number of a graph. We begin with some properties of the NLC-number depending on the diameter of the graph.

Proposition 3. Let $G$ be a connected graph of order $n \geq 3$.

(1) If $\operatorname{diam}(G) \leq 2$, then $\chi_{L}(G)=\chi_{N L}(G)$.

(2) If $\operatorname{diam}(G) \geq 4$, then $\chi_{N L}(G) \leq n-2$.

Proof. (1) Let $\Pi=\left\{S_{1}, \ldots, S_{k}\right\}$ be an ML-coloring of $G$. If $\operatorname{diam}(G)=2$, then for every $x \in V$ we have $\min \left\{2, d\left(x, S_{i}\right)\right\}=d\left(x, S_{i}\right)$. Hence, $n r(x \mid \Pi)=r(x \mid \Pi)$, for every $x \in V(G)$, that is, $\Pi$ is also an NL-coloring of $G$. Thus, $\chi_{N L}(G) \leq \chi_{L}(G)$, and by Proposition 1, we have $\chi_{N L}(G)=\chi_{L}(G)$.

(2) If $\operatorname{diam}(G) \geq 4$, then there exist vertices $u, v \in V(G)$ such that $d(u, v)=4$. Take $x_{1}, x_{2}, x_{3} \in V(G)$ such that the set $\left\{u, x_{1}, x_{2}, x_{3}, v\right\}$ induces a shortest path joining vertices $u$ and $v$, and $d\left(u, x_{3}\right)=d\left(u, x_{2}\right)+1=d\left(u, x_{1}\right)+2=3$. The partition $\Pi=\left\{S_{1}, S_{2}, S_{3}\right\} \cup\left\{\{z\}: z \notin S_{1} \cup S_{2} \cup S_{3}\right\}$, where $S_{1}=\left\{u, x_{3}\right\}, S_{2}=\left\{v, x_{1}\right\}$ and $S_{3}=\left\{x_{2}\right\}$, is clearly a coloring of $G$. We claim that $\Pi$ is also an NL-coloring. Indeed, $n r(u \mid \Pi)=(0,1,2, \ldots) \neq(0,1,1, \ldots)=n r\left(x_{3} \mid \Pi\right)$ and $n r(v \mid \Pi)=(1,0,2, \ldots) \neq$ $(1,0,1, \ldots)=n r\left(x_{1} \mid \Pi\right)$. Hence, $\chi_{N L}(G) \leq n-2$.

Theorem 1. Let $G$ be a non-trivial graph of order $n$ and maximum degree $\Delta$. If $\chi_{N L}(G)=k$ and $\Delta \leq k-1$, then

(1) $n \leq k 2^{k-1} \quad$ and $\quad(2) n \leq k \sum_{j=0}^{\Delta}\left(\begin{array}{c}k-1 \\ j\end{array}\right)$.

In addition, if $G$ has no isolated vertices, then:

(3) $n \leq k\left(2^{k-1}-1\right) \quad$ and $\quad(4) n \leq k \sum_{j=1}^{\Delta}\left(\begin{array}{c}k-1 \\ j\end{array}\right)$.

Proof. Let $\Pi=\left\{S_{1}, \ldots, S_{k}\right\}$ be a k-NL-coloring of $G$. An upper bound of the order of $G$ is given by the maximum number of suitable $k$-tuples $\operatorname{nr}(x \mid \Pi), x \in V(G)$.

(1) There are at most $2^{k-1}$ possible tuples with the $i$-th component equal to 0 and the remaining components equal to 1 or 2 . Therefore, $\left|S_{i}\right| \leq 2^{k-1}$. Hence, $n=|V(G)|=$ $\sum_{i=1}^{k}\left|S_{i}\right| \leq \sum_{i=1}^{k} 2^{k-1}=k 2^{k-1}$.

(2) If $\Delta \leq k-1$ and $x \in S_{i}$, then the $i$-th component of the $k$-tuple $n r(x \mid \Pi)$ is 0 and at most $\Delta$ components are equal to 1 . Therefore, $\left|S_{i}\right| \leq \sum_{j=0}^{\Delta}\left(\begin{array}{c}k-1 \\ j\end{array}\right)$, and the upper bound follows.

(3) In this case, for every $x \in V(G)$, the k-tuple $n r(x \mid \Pi)$ has at least one component equal to 1 . There are $2^{k-1}-1 k$-tuples with the $i$-th component equal to 0 and the remaining components equal to 1 or 2 , but not all them equal to 2 . Hence, $n=|V(G)|=$ $\sum_{i=1}^{k}\left|S_{i}\right| \leq \sum_{i=1}^{k}\left(2^{k-1}-1\right)=k\left(2^{k-1}-1\right)$. 
(4) If $\Delta \leq k-1$ and $G$ has no isolated vertices, then, for every $x \in S_{i}$, the $i$-th component of the $k$-tuple $n r(x \mid \Pi)$ is 0 , and the number of components which are equal to 1 is at least 1 and at most $\Delta$. Therefore, $\left|S_{i}\right| \leq \sum_{j=1}^{\Delta}\left(\begin{array}{c}k-1 \\ j\end{array}\right)$, and the upper bound follows.

Notice that the bounds displayed in items (3) and (4) of Theorem 1 apply also for connected graphs.

Next, for every integer $k \geq 3$, we build a connected graph $G_{k}=\left(V_{k}, E_{k}\right)$ of maximum order with NLC-number $k$. The set $V_{k}$ of vertices of $G_{k}$ is the set of all words of length $k$ in the alphabet $\{0,1,2\}$ having exactly one 0 and at least one 1 . To define the edges of $G_{k}$, let $W_{i}$ be the set of words $x_{1} \ldots x_{k} \in V_{k}$ such that $x_{i}=0$, for every $i \in\{1, \ldots, k\}$, so that $\left\{W_{1}, \ldots, W_{k}\right\}$ is a partition of $V_{k}$. For every $x, y \in V_{k}$, if $x=x_{1} \ldots x_{k} \in W_{i}$ and $y=y_{1} \ldots y_{k} \in W_{j}$, then $x y \in E_{k}$ if and only if $i \neq j, x_{j}=1$ and $y_{i}=1$ (see an illustration of graph $G_{3}$ in Figure 2).
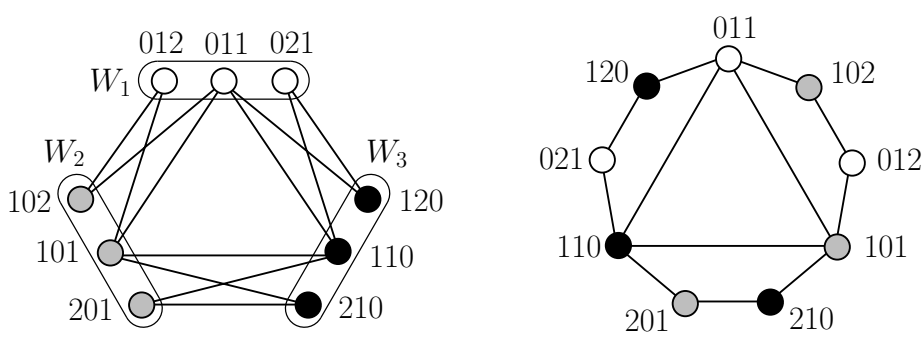

Figure 2: Two representations of the graph $G_{3}$.

Let $n_{k}=k \cdot\left(2^{k-1}-1\right), m_{k}=k \cdot(k-1) \cdot 2^{2 k-5}, \delta_{k}=2^{k-2}$ and $\Delta_{k}=(k-1) \cdot 2^{k-2}$.

Proposition 4. For every integer $k \geq 3, G_{k}$ is a connected graph of order $n_{k}$, size $m_{k}$, diameter 3 , minimum degree $\delta_{k}$, maximum degree $\Delta_{k}$ such that $\chi_{N L}\left(G_{k}\right)=k$.

Proof. For every $i \in\{1, \ldots, k\}$, we have $\left|W_{i}\right|=2^{k-1}-1$ because there are $2^{k-1}$ words in the alphabet $\{0,1,2\}$ with exactly one 0 in the position $i$, and only one of these words has no 1 's. Hence, $\left|V_{k}\right|=k \cdot\left(2^{k-1}-1\right)=n_{k}$.

Let $x \in W_{1}$ and $y \in W_{2}$. Notice that $x y \in E_{k}$ if and only if $x=01 x_{3} \ldots x_{k}$ and $y=10 y_{3} \ldots y_{k}$. Hence, the number of edges with an endpoint in $W_{1}$ and the other in $W_{2}$ is $\left(2^{k-2}\right)^{2}=2^{2 k-4}$. By symmetry, the number of edges with an endpoint in $W_{i}$ and the other in $W_{j}$ is $2^{2 k-4}$ for every pair $i, j \in\{1, \ldots, k\}$ with $i \neq j$. Since the sets $W_{i}$ are independent in $G_{k}$, we have $\left|E_{k}\right|=\left(\begin{array}{c}k \\ 2\end{array}\right) 2^{2 k-4}=k \cdot(k-1) \cdot 2^{2 k-5}=m_{k}$.

Now, let $x \in W_{i}$. If $x_{j}=1$, then $x$ is adjacent to every vertex $y \in W_{j}$ such that $y_{i}=1$. There are $2^{k-2}$ such vertices in $W_{j}$. Thus, $\operatorname{deg}(x)=\left|\left\{j: x_{j}=1\right\}\right| 2^{k-2}$. Hence, the minimum degree is attained by the vertices $x=x_{1} \ldots x_{k} \in V_{k}$ such that $\left|\left\{j: x_{j}=1\right\}\right|=1$, whereas the maximum degree is attained when $\left|\left\{j: x_{j}=1\right\}\right|=k-1$. Therefore, we have $\delta_{k}=2^{k-2}$ and $\Delta_{k}=(k-1) 2^{k-2}$.

Let $k \geq 3$. To prove that $G_{k}$ has diameter 3 , we show that the distance between any two different vertices $x, y \in V_{k}$ is at most 3 and at least two of them are at distance 3 . We distinguish two cases.

If $x, y \in W_{i}$ for some $i \in\{1, \ldots, k\}$, then we may assume without loss of generality that $x=0 x_{2} \ldots x_{k} \in W_{1}$ and $y=0 y_{2} \ldots y_{k} \in W_{1}$. Observe that $d(x, y)=2$ if and only if $x$ and $y$ 
have a common neighbor and, by definition of $G_{k}$, this happens if and only if $x_{j}=y_{j}=1$ for some $j \in\{2, \ldots, k\}$. If this condition does not hold, then $x_{h}=y_{l}=1$ and $x_{l}=y_{h}=2$, for some $h, l \in\{2, \ldots, k\}$. Without loss of generality, we may assume $h=2$ and $l=3$. In such a case, $x=012 x_{4} \ldots x_{k}, y=021 y_{4} \ldots y_{k}$, and $d(x, y)=3$ because:

$$
x=012 x_{4} \ldots x_{k} \sim 101 y_{4} \ldots y_{k} \sim 110 y_{4} \ldots y_{k} \sim 021 y_{4} \ldots y_{k}=y .
$$

If $x \in W_{i}$ and $y \in W_{j}$ for some $i, j \in\{1, \ldots, k\}, i \neq j$, then we may assume by symmetry that $x=0 x_{2} x_{3} \ldots x_{k} \in W_{1}$ and $y=y_{1} 0 y_{3} \ldots y_{k} \in W_{2}$. If $x_{2}=y_{1}=1$, then $x y \in E_{k}$ and $d(x, y)=1$. If $x_{2}=1$ and $y_{1}=2$, then $y_{l}=1$ for some $l \in\{3, \ldots, k\}$, and $d(x, y) \leq 3$ since:

$$
x=01 x_{3} \ldots x_{k} \sim 10 y_{3} \ldots{ }_{1}^{l} \ldots y_{k} \sim 21 y_{3} \ldots{ }^{l)} \ldots y_{k} \sim 20 y_{3} \ldots 1 \ldots y_{k}=y .
$$

In a similar way, we can prove that $d(x, y) \leq 3$ if $x_{2}=2$ and $y_{1}=1$. It only remains to consider the case $x_{2}=y_{1}=2$. If $x_{l}=y_{l}=1$ for some $l \in\{3, \ldots, k\}$, then $d(x, y) \leq 2$ because:

$$
x=02 x_{3} \ldots \stackrel{l}{1} \ldots x_{k} \sim 11 y_{3} \ldots \stackrel{l)}{0} \ldots y_{k} \sim 20 y_{3} \ldots \stackrel{l}{1} \ldots y_{k}=y .
$$

Otherwise, $k \geq 4$ and there exist $h, l \in\{3, \ldots, k\}$ such that $x_{h}=y_{l}=1$ and $x_{l}=y_{h}=2$. We may assume without loss of generality that $h=3$ and $l=4$. Then, $d(x, y) \leq 3$ since

$$
x=0212 x_{5} \ldots x_{k} \sim 1101 y_{5} \ldots y_{k} \sim 1110 y_{5} \ldots y_{k} \sim 2021 y_{5} \ldots y_{k}
$$

Finally, to see that $\chi_{N L}\left(G_{k}\right)=k$, observe first that, according to Theorem 1 (3), since $G_{k}$ is a connected graph of order $\left|V_{k}\right|=n_{k}=k \cdot\left(2^{k-1}-1\right)>(k-1)\left(2^{(k-1)-1}-1\right)$, we have $\chi_{N L}\left(G_{k}\right) \geq k$. Besides, $\left\{W_{1}, \ldots, W_{k}\right\}$ is clearly an NL-coloring of $G_{k}$. Indeed, $W_{1}, \ldots, W_{k}$ are independent sets. Moreover, if $x$ and $y$ are two different vertices of $W_{i}$, then $x_{j} \neq y_{j}$ for some $j \neq i$. Hence, $\left\{x_{j}, y_{j}\right\}=\{1,2\}$. We may assume without loss of generality that $x_{j}=1$ and $y_{j}=2$. Then, $x$ has a neighbor in $W_{j}$, but $y$ has no neighbor in $W_{j}$. Therefore, $\left\{W_{1}, \ldots, W_{k}\right\}$ is an NL-coloring, implying that $\chi_{N L}\left(G_{k}\right)=k$.

Corollary 1. The bounds displayed in items (1) and (3) of Theorem 1 are tight, for every $k \geq 3$.

Proof. For every $k \geq 3$, the graph $G_{k}$ attains the bound given in Theorem 1 (3). If we add $k$ isolated vertices to $G_{k}$, then we have a graph attaining the bound given in Theorem 1 (1).

Proposition 5. Let $k \geq 3$ and $\mu_{k}=k(k-1) 2^{k-3}$. If $H$ is a graph with no isolated vertices such that $\chi_{N L}(H)=k$, then

(1) $H$ is isomorphic to a subgraph of $G_{k}$.

(2) If $H$ has order $n_{k}$, then $\mu_{k} \leq|E(H)| \leq m_{k}$.

Proof. Let $\Pi=\left\{S_{1}, \ldots, S_{k}\right\}$ be an NL-partition of $H$. Recall that if $x \in V(H)$, then the tuple $n r(x \mid \Pi)=\left(x_{1}, \ldots, x_{k}\right)$ satisfies $x_{i}=0$ if $x \in S_{i}$, and $x_{j} \in\{1,2\}$, if $j \neq i$. Moreover, $x_{j}=1$ for some $j \neq i$, since $H$ has no isolated vertices.

To prove item (1), we identify $x \in V(H)$ with vertex $x_{1} \ldots x_{k} \in V\left(G_{k}\right)$, whenever $n r(x \mid \Pi)=\left(x_{1}, \ldots, x_{k}\right)$. If $x y \in E(H)$, then $x \in S_{i}$ and $y \in S_{j}$, for some $i, j \in\{1, \ldots, k\}$ with $i \neq j$. Thus, if $\operatorname{nr}(x \mid \Pi)=\left(x_{1}, \ldots, x_{k}\right)$ and $\operatorname{nr}(y \mid \Pi)=\left(y_{1}, \ldots, y_{k}\right)$, then we have $x_{i}=y_{j}=0$ 
and $x_{j}=y_{i}=1$, that is, $x_{1} \ldots x_{k}$ and $y_{1} \ldots y_{k}$ are adjacent in $G_{k}$. Hence, $H$ is isomorphic to a subgraph of $G_{k}$.

Since $H$ is isomorphic to a subgraph of $G_{k}$, we have $|E(H)| \leq E\left(G_{k}\right) \leq m_{k}$. Hence, the upper bound of item (2) holds. To prove the lower bound, notice that if $|V(H)|=|V(G)|=$ $n_{k}$, then $S_{i}$ has $2^{k-1}-1$ vertices for every $i \in\{1, \ldots, k\}$ and $2^{k-2}$ of them are adjacent to a vertex of $S_{j}$, if $j \neq i$. Hence, fixed $i, j \in\{1, \ldots, k\}$ with $i \neq j$, the number of edges with an endpoint in $S_{i}$ and the other in $S_{j}$ is at least $2^{k-2}$. Therefore, $|E(H)| \geq\left(\begin{array}{l}k \\ 2\end{array}\right) 2^{k-2}=$ $k(k-1) 2^{k-3}=\mu_{k}$.

Remark 5. As a consequence of Proposition 5, if $H$ is a graph of order at most $n_{k}$ without isolated vertices not isomorphic to any subgraph of $G_{k}$, then $\chi_{N L}(H) \geq k+1$. However, the converse is not true: there are subgraphs of $G_{k}$ with NLC-number greater than $k$. For example, the cycle of order $4, C_{4}$, is a subgraph of $G_{3}$, but $\chi_{N L}\left(C_{4}\right)=4$.

Next, we relate the NLC-number $\chi_{N L}(G)$ to the independence number $\alpha(G)$ of a twin-free graph $G$.

Proposition 6. If $G$ is a twin-free graph of order $n$, then $\chi_{N L}(G) \leq n-\alpha(G)+1$. Moreover, this bound is tight.

Proof. Let $\Omega$ be a maximum independent set of $G$, that is, an independent set such that $|\Omega|=\alpha(G)$. Consider the partition $\Pi=\{\Omega\} \cup\{\{z\}: z \in V(G) \backslash \Omega\}$. Notice that, since $G$ is a twin-free graph, the partition $\Pi$ is an NL-coloring of $G$. As $|\Pi|=n-\alpha(G)+1$, $\chi_{N L}(G) \leq n-\alpha(G)+1$.

To prove the tightness of the bound, let $H$ be the graph obtained from the complete graph $K_{r}$ by attaching $r-1$ leaves to respectively $r-1$ different vertices of $K_{r}$. Then, $H$ is a connected twin-free graph of order $2 r-1$, such that $\alpha(H)=r$. Besides, $\chi_{N L}(H) \geq \chi(H)=r$ and it is easy to check that $\chi_{N L}(H)=r$. Indeed, any partition with all parts but one of size two, such that each part of size two contains a leaf $u$ together with a vertex of $K_{r}$ non-adjacent to $u$, is an r-NL-coloring of $H$. Hence, $\chi_{N L}(H)=r=(2 r-1)-r+1=|V(H)|-\alpha(H)+1$. 


\section{Extremal graphs}

In this section, we focus our attention on graphs with NLC-number close to the order. In $[11,12]$, all connected graphs of order $n$ and $\chi_{L}(G)=n$ and $\chi_{L}(G)=n-1$ were characterized. Now, we approach the same problems for $\chi_{N L}(G)$. In fact, we show that the graphs achieving these extreme values are the same for both parameters.

Theorem 2 ([11]). If $G$ is a connected graph of order $n \geq 3$, then $\chi_{L}(G)=n$ if and only if $G$ is a complete multipartite graph.

Notice that complete graphs $K_{n}$, complete bipartite graphs $K_{h, n-h} \cong \overline{K_{h}} \vee \overline{K_{n-h}}$, stars $S_{1, n-1} \cong \overline{K_{1}} \vee \overline{K_{n-1}}$ and complete split graphs $\overline{K_{h}} \vee K_{n-h}$ are some examples of complete multipartite graphs.

Theorem 3. If $G$ is a graph of order $n \geq 3$, then $\chi_{N L}(G)=n$ if and only if $G$ is either a complete multipartite graph or $G=\overline{K_{n}}$.

Proof. Clearly, $\chi_{N L}\left(\overline{K_{n}}\right)=n$. By other hand, if $G$ is a complete multipartite graph, then according to Theorem 2, $\chi_{L}(G)=n$. Thus, by Proposition $1, \chi_{N L}(G)=n$.

Conversely, we distinguish two cases depending of whether or not the graph $G$ is connected.

If $G$ is a connected graph of order $n \geq 3$ which is not a complete multipartite graph, then there exists a pair of non-adjacent vertices $u, v \in V(G)$ such that $N(u) \neq N(v)$. Let $\Pi=\left\{S_{1}, S_{2}, \ldots, S_{n-1}\right\}$ be the coloring of $G$ such that $S_{1}=\{u, v\}$ and, for every $i \neq 1$, $\left|S_{i}\right|=1$. Certainly, $\Pi$ is an NL-coloring, since $N(u) \neq N(v)$. Without loss of generality, we can consider $z \in V(G)$ such that $u z \in E(G)$ and $v z \notin E(G)$, so $d(u,\{z\}) \neq d(v,\{z\})$ and $n r(u \mid \Pi) \neq n r(v \mid \Pi)$, and thus $\chi_{N L}(G) \leq n-1$.

If $G$ is a non-connected graph of order $n \geq 3$ other than $\overline{K_{n}}$, then there exists a pair of adjacent vertices $x, y \in V(G)$ and there exist a vertex $t$ in a connected component different from that of $x$ and $y$. Let $\Pi=\left\{S_{1}, S_{2}, \ldots, S_{n-1}\right\}$ be the coloring of $G$ such that $S_{1}=\{x, t\}$ and, for every $i \neq 1,\left|S_{i}\right|=1$. Clearly, $\Pi$ is an NL-coloring, since $n r(x \mid \Pi) \neq n r(t \mid \Pi)$, and thus $\chi_{N L}(G) \leq n-1$.

We next study the graphs of order $n$ and NLC-number $n-1$. For this, we introduce first some families of graphs that will play an important role.

- Let $\mathcal{H}$ denote the set of all connected graphs $G$ of order $n \geq 3$ such that, for some vertex $v \in G, G-v$ is a complete multipartite graph.

- For $G \in \mathcal{H}$, call $V_{1}, V_{2}, \ldots, V_{k}, k \geq 2$, to the partite sets of $G-v$; and let $n_{i}=\left|V_{i}\right|$ and $a_{i}=\left|N(v) \cap V_{i}\right|$ for $1 \leq i \leq k$.

- Let $\mathcal{F}$ denote the set of all graphs $G \in \mathcal{H}$ satisfying al least one of the following two properties:

(1) $a_{i} \in\left\{0, n_{i}\right\}$ for every $i \in\{1, \ldots, k\}$, and $\left|\left\{i \in\{1, \ldots, k\} \mid a_{i}=0\right\}\right| \geq 2$.

(2) There is exactly one integer $i \in\{1, \ldots, k\}$ such that $a_{i} \notin\left\{0, n_{i}\right\}$, and $a_{i}=n_{i}-1$ for this integer $i$. 
- Let $\mathcal{G}$ denote the set of all graphs of order $n$ that are the join of $2 K_{2}$ and a complete multipartite graph $G^{*}$ of order $n-4 \geq 1$, that is, $G=G^{*} \vee 2 K_{2}, V(G)=V_{1} \cup V_{2}$, $V_{1}=\left\{v_{1}, \ldots, v_{4}\right\}, V_{2}=\left\{v_{5}, \ldots, v_{n}\right\}, G\left[V_{1}\right]=2 K_{2}$ and $G\left[V_{2}\right]=G^{*}$ and all the edges that connect vertices of $V_{1}$ with vertices of $V_{2}$ are in $E(G)$ (see next section for more properties of join graphs).

Theorem 4 ([12]). If $G$ is a connected graph of order $n \geq 4$, then $\chi_{L}(G)=n-1$ if and only if $G \in \mathcal{F} \cup \mathcal{G}$.

Lemma 1. If $G$ is a graph of order $n \geq 5, \chi_{N L}(G)=n-1$ and $2 K_{2} \prec G$, then $G \in \mathcal{G}$.

Proof. Let $S=\left\{a_{1}, a_{2}, b_{1}, b_{2}\right\} \subset V(G)$ be a set such that $a_{1} a_{2}, b_{1} b_{2} \in E(G)$ and $G[S] \cong 2 K_{2}$. Let $w \in V(G) \backslash S$ and let $h=|N(w) \cap S|$. Suppose that $h \leq 3$ and $w b_{2} \notin E(G)$. Consider the $(n-2)$-partition $\Pi=\left\{S_{1}, S_{2}, S_{3}, \ldots, S_{n-2}\right\}$, where $S_{1}=\left\{w, b_{2}\right\}, S_{2}=\left\{a_{1}, b_{1}\right\}$ and $S_{3}=\left\{a_{2}\right\}$. Notice that $\Pi$ is an NL-coloring of $G$, except in two cases: that $w a_{2} \notin E(G)$ and also $w$ is adjacent to $a_{1}$ or to $b_{1}$. In the case that $w a_{2} \notin E(G)$ and $w a_{1} \in E(G)$ we take $S_{1}=\left\{w, b_{2}\right\}, S_{2}=\left\{a_{2}, b_{1}\right\}$ and $S_{3}=\left\{a_{1}\right\}$. If $w a_{2} \notin E(G)$ and $w b_{1} \in E(G)$ we take $S_{1}=\left\{w, a_{2}\right\}, S_{2}=\left\{a_{1}, b_{2}\right\}$ and $S_{3}=\left\{b_{1}\right\}$. Thus, $\chi_{N L}(G) \leq n-2$, a contradiction. So, we have proved that each vertex of $V(G) \backslash S$ is adjacent to every vertex of $S$.

Take $H=G[V(G) \backslash S]$. Suppose that $H$ is not a complete multipartite graph. Let $u, v \in V(G) \backslash S$ such that $u v \notin E(G)$ and $N(u) \neq N(v)$. Consider the $(n-2)$-partition $\Pi=\left\{S_{1}, S_{2}, S_{3}, \ldots, S_{n-2}\right\}$, where $S_{1}=\{u, v\}$ and $S_{2}=\left\{a_{1}, b_{1}\right\}$. Certainly, $\Pi=$ is an NLcoloring of $G$, i.e., $\chi_{N L}(G) \leq n-2$, a contradiction. Hence, $H$ is a complete multipartite graph.

Lemma $2([12])$. If $G$ is a connected graph of order $n \geq 4$ with $\chi_{L}(G)=n-1$ and $2 K_{2} \nprec G$, then $G \in \mathcal{F}$.

Lemma 3. Let $G$ be a graph of order $n \geq 5$. Let $S=\left\{u_{1}, u_{2}, u_{3}, v\right\}$ be a set of vertices of $V(G)$ such that $d\left(u_{1}, u_{3}\right)=2, u_{2} \in N\left(u_{1}\right) \cap N\left(u_{3}\right)$ and $N\left(u_{1}\right) \neq N\left(u_{3}\right)$. If $S$ induces a subgraph of $G$ isomorphic to $P_{3}+K_{1}$, then $\chi_{N L}(G) \leq n-2$.

Proof. Let $w$ be a vertex of $N\left(u_{1}\right) \cup N\left(u_{3}\right)$ not belonging to $N\left(u_{1}\right) \cap N\left(u_{3}\right)$. Take the partition $\Pi=\left\{S_{1}, S_{2}, S_{3}, \ldots, S_{n-2}\right\}$ such that $S_{1}=\left\{u_{1}, u_{3}\right\}, S_{2}=\left\{u_{2}, v\right\}$ and $S_{3}=\{w\}$. Notice that $d\left(u_{1}, S_{3}\right)=1<d\left(u_{3}, S_{3}\right)$ and $d\left(u_{2}, S_{1}\right)=1<d\left(v, S_{1}\right)$. Hence, $\Pi$ is an NL-coloring of $G$.

Lemma 4. Let $G$ be a graph of order $n \geq 5$, diameter 3 such that $2 K_{2} \nprec G$. If $\chi_{N L}(G)=$ $n-1$, then $\chi_{L}(G)=n-1$.

Proof. Suppose on the contrary that there exist a graph $G$ such that $\chi_{N L}(G)=n-1$ and $\chi_{L}(G) \leq n-2$. Let $\Pi=\left\{S_{1}, S_{2}, \ldots S_{n-2}\right\}$ be an ML-coloring of cardinality $n-2$. $\Pi$ can not be an NL-coloring. If $S_{1}=\left\{u_{1}, v_{1}\right\}, S_{2}=\left\{u_{2}, v_{2}\right\}, S_{3}=\left\{w_{3}\right\}, \ldots, S_{n-2}=\left\{w_{n-2}\right\}$, then we can assume without loss of generality that, for every $j \in\{2, \ldots, n-2\}, \operatorname{nr}\left(u_{1} \mid \Pi\right)=$ $n r\left(v_{1} \mid \Pi\right)$. This means that, for every $j \in\{2, \ldots, n-2\}$, either $d\left(u_{1}, S_{j}\right)=d\left(v_{1}, S_{j}\right)=1$ or $2 \leq d\left(u_{1}, S_{j}\right), d\left(v_{1}, S_{j}\right) \leq 3$. Notice that if, for every $j \in\{3, \ldots, n-2\}$, we have $d\left(u_{1}, S_{j}\right)=$ $d\left(v_{1}, S_{j}\right)=1$, then $1 \leq d\left(u_{1}, S_{j}\right), d\left(v_{1}, S_{j}\right) \leq 2$. Hence, we can suppose without loss of generality that $d\left(u_{1}, w_{3}\right)=2$ and $d\left(v_{1}, w_{3}\right)=3$. Let $z \in V(G)$ be such that $d\left(u_{1}, z\right)=$ $d\left(z, w_{3}\right)=1$. If, for some $j \in\{4, \ldots, n-2\}$, we have $z=w_{j}$, then $d\left(v_{1}, z\right)=1$, and thus $d\left(v_{1}, w_{3}\right)=2$, a contradiction. So, we can suppose without loss of generality that $z=u_{2}$. Let $x \in V(G)$ be such that $d\left(v_{1}, x\right)=1$ and $d\left(x, w_{3}\right)=2$. Notice that $x=v_{2}$, as otherwise, if for 
some $j \in\{4, \ldots, n-2\}, x=w_{j}$, then $d\left(u_{1}, x\right)=1$, and according to Lemma $3, d\left(x, w_{3}\right)=1$ since $N\left(u_{1}\right)=N\left(w_{3}\right)$, a contradiction. Hence, the subgraph induced by $\left\{u_{2}, w_{3}, v_{1}, v_{2}\right\}$ is isomorphic to $2 K_{2}$, which is again a contradiction.

Theorem 5. Let $G$ be a graph of order $n \geq 5$. Then, $\chi_{N L}(G)=n-1$ if and only if either $G \in \mathcal{F} \cup \mathcal{G}$ or $G \cong H+K_{1}$, where $H$ is an arbitrary complete multipartite graph.

Proof. If $G \in \mathcal{F} \cup \mathcal{G}$, then, according to Theorem 4, $\chi_{L}(G)=n-1$. This means that $\chi_{N L}(G) \geq n-1$, since $\chi_{L}(G) \leq \chi_{N L}(G)$. Hence, from Theorem 2 , we derive that $\chi_{N L}(G)=$ $n-1$.

Let $H$ be a complete bipartite graph of order $n-1$. According to Theorem $3, \chi_{N L}(H)=$ $n-1$. Let $G \cong H+K_{1}$ such that $V\left(K_{1}\right)=\{u\}$. If $v \in V(H)$, then it is straightforward to check that the $(n-1)$-coloring of $G \Pi=\left\{S_{1}, \ldots, S_{n-1}\right\}$ such that $S_{1}=\{u, v\}$, is an NL-coloring of $G$. Thus, $\chi_{N L}(G)=n-1$.

Conversely, let $G$ be a graph such that $\chi_{N L}(G)=n-1$. We distinguish two case depending on whether or not the graph $G$ is connected.

Suppose that $G$ is a connected graph. By Theorem 3 and Proposition 3, it follows that $2 \leq \operatorname{diam}(G) \leq 3$. If either $\operatorname{diam}(\mathrm{G})=2$ or $2 K_{2} \prec G$, then according to Proposition 3 , Theorem 4 and Lemma 1, we derive that $G \in \mathcal{F} \cup \mathcal{G}$. If $G$ is a graph of diameter $\operatorname{diam}(\mathrm{G})=3$ such that $2 K_{2} \nprec G$, then from Lemma 4 and Lemma 2 , it follows that $G \in \mathcal{F}$.

Assume that $G$ is a non-connected graph. We distinguish cases depending on the connected components of $G$.

Case 1. All components of $G$ have at least two vertices. Let $C_{1}, C_{2}$ a pair of components of $G$ such that $\left|C_{1}\right| \geq 3$. Take $u_{1}, v_{1}, w_{1} \in C_{1}$ and $u_{2}, w_{2} \in C_{2}$ such that $u_{1} v_{1}, v_{1} w_{1} \in E(G)$. Then, it is straightforward to check that the $(n-2)$-coloring $\Pi=\left\{S_{1}, \ldots, S_{n-2}\right\}$ such that $S_{1}=\left\{u_{1}, u_{2}\right\}, S_{2}=\left\{v_{1}\right\}$ and $S_{3}=\left\{w_{1}, w_{2}\right\}$, is an NL-coloring. Thus, $\chi_{N L}(G) \leq n-2$.

Case 2. $G$ has at least two trivial components, i.e., $G$ contains at least two isolated vertices $u$ and $v$. Let $C_{1}, C_{2}, C_{3}$ be three components of $G$ such that $C_{1}=\{x\}, C_{2}=\{y\}$ and $z, w \in C_{3}$. Then, it is straightforward to check that the $(n-2)$-coloring $\Pi=\left\{S_{1}, \ldots, S_{n-2}\right\}$ such that $S_{1}=\{x, z\}$ and $S_{2}=\{y, w\}$, is an NL-coloring. Thus, $\chi_{N L}(G) \leq n-2$.

Case 3. $G$ contains exactly one isolated vertex $u$. Let $H$ be the graph of order $n-1$ without isolated vertices, such that $G \cong H+K_{1}$ and $V\left(K_{1}\right)=\{u\}$. Observe that $\chi_{N L}(H)=$ $\chi_{N L}(G)=n-1$, since if $\Pi=\left\{S_{1}, S_{2}, \ldots, S_{k}\right\}$ is a $k$-NL-coloring of $H$, then the $k$-coloring $\Pi=\left\{S_{1}^{\prime}, S_{2}, \ldots, S_{k}\right\}$ where $S_{1}^{\prime}=S_{1} \cup\{u\}$, is an NL-coloring of $G$. Thus, according to Theorem $3, H$ is a complete multipartite graph. 


\section{$5 \quad$ Join and disjoint union}

This section is devoted to analysing the behavior of the NLC-number with respect to two graph operations: join and disjoint union.

A graph $G=(V, E)$ is a join graph if it is the join $G_{1} \vee G_{2}$ of two graphs $G_{1}=\left(V_{1}, E_{1}\right)$ and $G_{2}=\left(V_{2}, E_{2}\right)$, i.e., if $V=V_{1} \cup V_{2}$ and $E=E_{1} \cup E_{2} \cup E^{\prime}$, where $E^{\prime}=\left\{v_{1} v_{2}: v_{1} \in V_{1}, v_{2} \in V_{2}\right\}$.

Some examples of graphs obtained as the join of two graphs are the fan $F_{n}=K_{1} \vee P_{n-1}$, the wheel $W_{n}=K_{1} \vee C_{n-1}$ and the complete bipartite graph $K_{h, k}=\overline{K_{h}} \vee \overline{K_{k}}$.

Clearly, $\chi\left(G_{1} \vee G_{2}\right)=\chi\left(G_{1}\right)+\chi\left(G_{2}\right)$. In [3], it is shown that, if $G_{1}$ and $G_{2}$ are two connected graphs of diameter at most two, then $\chi_{L}\left(G_{1} \vee G_{2}\right)=\chi_{L}\left(G_{1}\right)+\chi_{L}\left(G_{2}\right)$. But, in general, $\chi_{L}\left(G_{1} \vee G_{2}\right) \geq \chi_{L}\left(G_{1}\right)+\chi_{L}\left(G_{2}\right)$. For example, $\chi_{L}\left(P_{10}\right)=3$ and $\chi_{L}\left(P_{10} \vee P_{10}\right)=8$ $($ see $[3])$.

Next, we study the NLC-number of the join of two graphs.

Remark 6. As a straightforward consequence of the definition, the following properties hold. If $G_{1}, G_{2}$ and $G_{3}$ are three graphs, then

1. $G_{1} \vee G_{2}$ is a connected graph of diameter at most 2 .

2. $G_{1} \vee\left(G_{2} \vee G_{3}\right) \cong\left(G_{1} \vee G_{2}\right) \vee G_{3}$.

Let $r, n_{1}, \ldots, n_{r}, n$ be integers such that $2 \leq r, 1 \leq n_{1} \leq \ldots \leq n_{r}$ and $n=n_{1}+\ldots n_{r}$. The complete $r$-partite graph $K_{n_{1}, \ldots, n_{r}}$ is the graph $\overline{K_{n_{1}}} \vee \ldots \vee \overline{K_{n_{r}}}$. In the previous section, we have shown that the NLC-number of a complete multipartite graph equals the order. Thus, $\chi_{N L}\left(\overline{K_{n_{1}}} \vee \ldots \vee \overline{K_{n_{r}}}\right)=\chi_{N L}\left(K_{n_{1}, \ldots, n_{r}}\right)=n_{1}+\ldots+n_{r}=\chi_{N L}\left(\overline{K_{n_{1}}}\right)+\ldots+\left(\overline{K_{n_{r}}}\right)$. Next theorem extends this result to the join of general graphs.

Theorem 6. For every pair of graphs $G_{1}$ and $G_{2}, \chi_{N L}\left(G_{1} \vee G_{2}\right)=\chi_{N L}\left(G_{1}\right)+\chi_{N L}\left(G_{2}\right)$.

Proof. If $\Pi_{1}=\left\{S_{1}, \ldots, S_{h}\right\}$ is an NL-coloring of $G_{1}$ and $\Pi_{2}=\left\{T_{1}, \ldots, T_{k}\right\}$ is an NL-coloring of $G_{2}$ then, clearly, $\left\{S_{1}, \ldots, S_{h}, T_{1}, \ldots, T_{k}\right\}$ is an NL-coloring of $G_{1} \vee G_{2}$.

Now, let $\Pi$ be an NL-coloring of $G_{1} \vee G_{2}$. Observe that, given a vertex $v \in V\left(G_{i}\right)$ and a part $S$ of $\Pi$ such that $v \in S$, then $S \subseteq V\left(G_{i}\right)(i \in\{1,2\})$. On the other hand, if $v \in V\left(G_{i}\right)$ and $S \in \Pi$ such that $S \subseteq V\left(G_{j}\right), i, j \in\{1,2\}$ and $i \neq j$, then $d(v, S)=1$. As a consequence, reordering if necessary, we can consider $\Pi=\left\{S_{1}, \ldots, S_{\ell}, S_{\ell+1}, \ldots, S_{t}\right\}$ so that $\left\{S_{1}, \ldots, S_{\ell}\right\}$ is an NL-coloring of $G_{1}$ and $\left\{S_{\ell+1}, \ldots, S_{t}\right\}$ is an NL-coloring of $G_{2}$.

The disjoint union of two vertex-disjoint graphs $G$ and $H$ is the graph denoted by $G+H$ whose vertex and edge sets are $V(G) \cup V(H)$ and $E(G) \cup E(H)$, respectively. Next, we present some properties relating $\chi_{N L}(G+H)$ to $\chi_{N L}(G)$ and $\chi_{N L}(H)$.

Theorem 7. Let $G, H$ be two graphs with $\chi_{N L}(G)=k$ and $\chi_{N L}(H)=h$. The following bounds hold for $\chi_{N L}(G+H)$ and are best possible.

(i) $\max \{h, k\} \leq \chi_{N L}(G+H)$.

(ii) If $G$ has exactly $k$ isolated vertices and $H$ has exactly $h$ isolated vertices, then $\chi_{N L}(G+$ $H)=k+h$;

(iii) in any other case, $\chi_{N L}(G+H) \leq k+h-1$. 
(iv) If $G$ contains a universal vertex, then $\chi_{N L}(G+G) \leq k+1$.

Proof. Any NL-coloring of $G+H$ induces an NL-coloring of $G$ and an NL-coloring of $H$, thus the first statement is true. For $k \geq 3$ and $h \leq k$, let $G$ and $H$ be the stars $S_{1, k-1}$ and $S_{1, h-1}$, respectively. Since $\chi_{N L}\left(S_{1, k-1}\right)=k, \chi_{N L}\left(S_{1, h-1}\right)=h$ and $\chi_{N L}\left(S_{1, k-1}+S_{1, h-1}\right)=k$, we have that the given bound is tight.

To prove (ii), notice that the union of an NL-coloring of $G$ and an NL-coloring of $H$ produces an NL-coloring of $G+H$, so $\chi_{N L}(G+H) \leq k+h$. On the other hand, since $G+H$ has $k+h$ isolated vertices, we have that $\chi_{N L}(G+H) \geq k+h$, and the equality follows.

In order to prove (iii), let $\Pi=\left\{S_{1}, \ldots, S_{k}\right\}$ and $\Pi^{\prime}=\left\{S_{1}^{\prime}, \ldots, S_{h}^{\prime}\right\}$ be NL-colorings of $G$ and $H$, respectively. Without loss of generality, we can assume that $G$ has less than $k$ isolated vertices, and, therefore, that $S_{1}$ contains no isolated vertices. In such a case, $\left\{S_{1} \cup S_{1}^{\prime}, S_{2}, \ldots, S_{k}, S_{2}^{\prime}, \ldots, S_{h}^{\prime}\right\}$ is an $(k+h-1)$-NL-coloring of $G+H$, establishing the desired bound. To see that this bound is tight, consider the case $\ell=k+h-1$ of proof of Theorem 8.

Finally, to prove item (iv), observe that from any given NL-coloring of $G$, we can obtain an NL-coloring of $G+G$ by painting the universal vertex of the second copy with a new color $k+1$, and painting any other vertex of the second copy with the same color as it has in the first copy. Since $\chi_{N L}\left(K_{k}+K_{k}\right) \leq k+1$, the bound is the best possible.

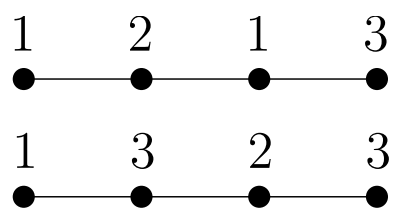

Figure 3: An NL-coloring of $2 P_{4}$.

Theorem 8. Let $h, k$ be integers such that $3 \leq h \leq k$. Then, for every $\ell \in[k, k+h]$, there exist graphs $G$ and $H$ such that $\chi_{N L}(G)=k, \chi_{N L}(H)=h$ and $\chi_{N L}(G+H)=\ell$.

Proof. For the case $\ell=k$, consider the stars $G \cong S_{1, k-1}$ and $H \cong S_{1, h-1}$. Then, it is easy to check that $\chi_{N L}\left(S_{1, k-1}+S_{1, h-1}\right)=\chi_{N L}\left(S_{1, k-1}\right)=k$.

Case $\ell \in[k+1, k+h-1]$. Let $G$ be a connected graph of order $k\left(2^{k-1}-1\right)$ with $\chi_{N L}(G)=k$ (take, for instance, the graph $G_{k}$ described in Section 3) and let $H$ be the graph obtained from the complete graph $K_{\ell-k}$ by hanging $h-1$ leaves to each of its vertices. Notice that $\chi_{N L}(H)=h$. Take an NL-coloring of $G$ with $k$ colors and notice that it is not possible to color any other vertex of $G+H$ with these colors. Let $\{1, \ldots, \ell-k\}$ be colors different from the previous ones and assign these colors to the vertices of $K_{\ell-k}$. Color with $\{1, \ldots, \ell-k\} \backslash\{i\}$ the leaves hanging from the vertex of $K_{\ell-k}$ with color $i$. In this way, we obtain an NL-coloring of $G+H$ with $k+(\ell-k)=\ell$ colors and it is not possible to do so with less colors. Then, $\chi_{N L}(G+H)=\ell$.

For the case $\ell=k+h$, take the empty graphs $G \cong \overline{K_{k}}$ and $H \cong \overline{K_{h}}$ and observe that $G+H \cong \overline{K_{k+h}}$ and $\chi_{N L}(G+H)=k+h$. 


\section{$6 \quad$ Split and Mycielski graphs}

This section is devoted to investigating the NLC-number in two important families of graphs: split graphs and Mycielski graphs.

A split graph is a graph such that the vertices can be partitioned into a clique and an independent set. When every vertex in the independent set is adjacent to every vertex in the clique it is said to be a complete split graph. Observe that complete split graphs are examples of complete multipartite graphs that we just studied in the previous section. We have taken a step further and we have studied the NLC-number of general connected split graphs.

For any connected split graph $G=(V, E)$ we can assume that there are two subsets $U$ and $W$ of $V$ such that

(i) $V=U \cup W, U \cap W=\emptyset$;

(ii) $G[U]$ is a complete graph;

(iii) $W$ is a maximal independent set, i.e., $W$ is an independent set that for each vertex $u \in U$, there exists a vertex $w \in W$ such that $u w \in E$.

For every $X \subseteq U$, we define $\mathcal{P}(X)=\{w \in W: N(w)=X\}$ and $\rho(G)=\max \{|X|+$ $|\mathcal{P}(X)|: X \subseteq U\}$. Observe that, by definition, $\rho(G) \geq|U|$.

Theorem 9. If $G$ is a connected split graph, then

$$
\chi_{N L}(G)=\left\{\begin{array}{lr}
\rho(G), & \text { if } \mathcal{P}(X)=\emptyset \text { for all } X \subseteq U \text { s.t. }|X|=|U|-1 \\
\max \{|U|+1, \rho(G)\}, & \text { if } \mathcal{P}(X) \neq \emptyset \text { for some } X \subseteq U \text { s.t. }|X|=|U|-1
\end{array}\right.
$$

Proof. First we will prove that $\chi_{N L}(G)$ is at least the given value. Assume $\chi_{N L}(G)=k$ and let $\Pi=\left\{S_{1}, \ldots, S_{k}\right\}$ be an NL-coloring of $G$. We say that $u$ has color $i$ if $u \in S_{i}$. Let $X \subseteq U$. The vertices in $\mathcal{P}(X)$ are pairwise twins and adjacent to all the vertices in $X$. Hence, different vertices of $X \cup \mathcal{P}(X)$ have different colors. Therefore,

$$
\chi_{N L}(G) \geq \max \{|X|+|\mathcal{P}(X)|: X \subseteq U\}=\rho(G) .
$$

In addition, if there is a set $X \subseteq U$ such that $|X|=|U|-1$ and $\mathcal{P}(X) \neq \emptyset$, we claim that $\chi_{N L}(G) \geq|U|+1$. Indeed, if $\rho(G) \geq|U|+1$, then the assertion is obvious. Otherwise, $\rho(G)=|U|$, which implies $\mathcal{P}(U)=\emptyset$ and $|\mathcal{P}(X)|=1$. Let $w \in W$ be the only vertex in $\mathcal{P}(X)$ and let $u \in U$ be the only vertex in $U \backslash X$. Since $N(u) \cap N(w)$ induces a complete graph of size $|U|-1$, we have that $\chi_{N L}(G) \geq|U|+1$.

Now we will prove that $\chi_{N L}(G)$ is at most the given value.

First, suppose that $\mathcal{P}(X)=\emptyset$ for all $X \subseteq U$ such that $|X|=|U|-1$. We construct an NL-coloring $\Pi=\left\{S_{1}, \ldots, S_{k}\right\}$, where

$$
k=\rho(G)=\max \{|X|+|\mathcal{P}(X)|: X \subseteq U\} .
$$

Let $U=\left\{x_{1}, \ldots, x_{r}\right\}$ and color each $x_{i}$ with $i$ for $1 \leq i \leq r$. Notice that this is possible since $k=\rho(G) \geq|U|$. Then, for every set $X \subseteq U$, color the elements of $\mathcal{P}(X)$ using $|\mathcal{P}(X)|$ distinct colors chosen from the ones not used to color the vertices of $X$. Notice that this is possible since $k-|X| \geq|\mathcal{P}(X)|$.

We claim that $\Pi$ is an NL-coloring. Indeed, two vertices with the same color are non adjacent by construction. Now, suppose that $u$ and $v$ have the same color $i$, for some $i \in$ 
$\{1, \ldots, k\}$. We prove that $u$ and $v$ are neighbor-located by $\Pi$. We consider two cases: either $u, v \in W$; or $u \in U$ and $v \in W$.

In the first case, to prove that $u$ and $v$ are neighbor-located by $\Pi$, it is enough to see that $N(u) \neq N(v)$. In fact, if $N(u)=N(v)=X$, then $u$ and $v$ belong to $\mathcal{P}(X)$, and thus $u$ and $v$ have different colors, contradicting the assumption.

In the second case, assume that $v \in \mathcal{P}(X)$. Then, $u \notin X=N(v)$, and so, $|X| \leq|U|-2$. The neighbors of $v \in \mathcal{P}(X)$ are colored with exactly $|X|$ different colors, with $|X| \leq|U|-2$. However, the neighbors of $u$ are colored with at least $|U|-1$ different colors. Hence, $u$ and $v$ are neighbor-located by $\Pi$.

Now, suppose that $\mathcal{P}(X) \neq \emptyset$ for some subset $X \subseteq U$ such that $|X|=|U|-1$. We construct an NL-coloring $\Pi=\left\{S_{1}, \ldots, S_{k}\right\}$, where $k=\max \{\rho(G),|U|+1\}$.

As before, let $U=\left\{x_{1}, \ldots, x_{r}\right\}$ and color each $x_{i}$ with $i$ for $1 \leq i \leq r$. Again, this is possible since $k \geq \rho(G) \geq|U|$.

Let $U^{\prime}=\{u \in U: \mathcal{P}(U \backslash\{u\}) \neq \emptyset\}$ and take a maximal twin-free subset $W^{\prime}$ of $N\left(U^{\prime}\right) \cap W$. Notice that, such a set exists and, by construction, $U^{\prime} \subseteq N\left(W^{\prime}\right)$. Color all the vertices of $W^{\prime}$ with color $r+1$. Finally, for every subset $X \subseteq U$, color the vertices in $\mathcal{P}(X) \backslash W^{\prime}$ with different colors chosen from the ones not used to color the vertices of $X$, and without using color $r+1$. Notice that it is possible since $k-|X| \geq|\mathcal{P}(X)|$.

We claim that $\Pi$ is an NL-coloring. Indeed, vertices with the same color are non adjacent by construction. Now suppose that $u$ and $v$ have the same color $i$, for some $i \in\{1, \ldots, k\}$. Without loss of generality, we may distinguish two cases: $u, v \in W$; or $u \in U$ and $v \in W$.

In the first case, $u \in \mathcal{P}\left(X_{u}\right)$ and $u \in \mathcal{P}\left(X_{v}\right)$, where $N(u)=X_{u} \neq N(v)=X_{v}$, for some $X_{u}, X_{v} \subseteq U$. Therefore, the set of colors of the neighbors of $u$ and $v$ are different. Hence, $u$ and $v$ are neighbor-located by $\Pi$.

In the second case, assume that $v \in \mathcal{P}(X)$. Then, $u \notin X=N(v)$, and so $|X| \leq|U|-1$. If $|X| \leq|U|-2$, the neighbors of $v \in \mathcal{P}(X)$ are colored with exactly $|X| \leq|U|-2$ different colors. However, the neighbors of $u$ are colored with at least $|U|-1$ different colors. Hence, $u$ and $v$ are neighbor-located by $\Pi$.

If $|X| \leq|U|-1$, the neighbors of $v \in \mathcal{P}(X)$ are colored with exactly $|X|$ different colors from $\{1, \ldots, r\}$ However, at least one neighbor of $u$ has color $r+1$. Hence, $u$ and $v$ are neighbor-located by $\Pi$.

Remark 7. The value of the NLC-number obtained for general split graphs fits with some known results, such as $\chi_{N L}\left(S_{1, n-1}\right)=n, \chi_{N L}\left(K_{n}\right)=n$, and $\chi_{N L}(G)=n$, whenever $G$ is a complete split graph.

Another interesting class of graphs are Mycielski graphs. Given a graph $G$ of order $n$, the Mycielski graph $\mu(G)$ of $G$ is a graph of order $2 n+1$ that contains $G$ as an induced subgraph. Concretely, if $V(G)=\left\{v_{1}, \ldots, v_{n}\right\}$, then $V(\mu(G))=\left\{v_{1}, \ldots, v_{n}\right\} \cup\left\{u_{1}, \ldots, u_{n}\right\} \cup\{w\}$ and $E(\mu(G))=E(G) \cup\left\{w u_{i}: 1 \leq i \leq n\right\} \cup\left\{v_{i} u_{j}: 1 \leq i, j \leq n, v_{i} v_{j} E(G)\right\}$ (see Figure 4). Thus, $|E(\mu(G))|=3|E(G)|+n$. From now on, we will use this terminology when referring to the set of vertices of a graph $G$ and its Mycielski graph $\mu(G)$.

Mycielski [32] designed these graphs to prove that it is possible to increase the chromatic number of a graph without increasing the clique number. More precisely, $\omega(\mu(G))=$ $\max (2, \omega(G))$ and $\chi(\mu(G))=\chi(G)+1$.

Next, we give a similar partial result for the NLC-number.

Proposition 7. For every graph $G, \chi_{N L}(\mu(G)) \leq \chi_{N L}(G)+1$. 

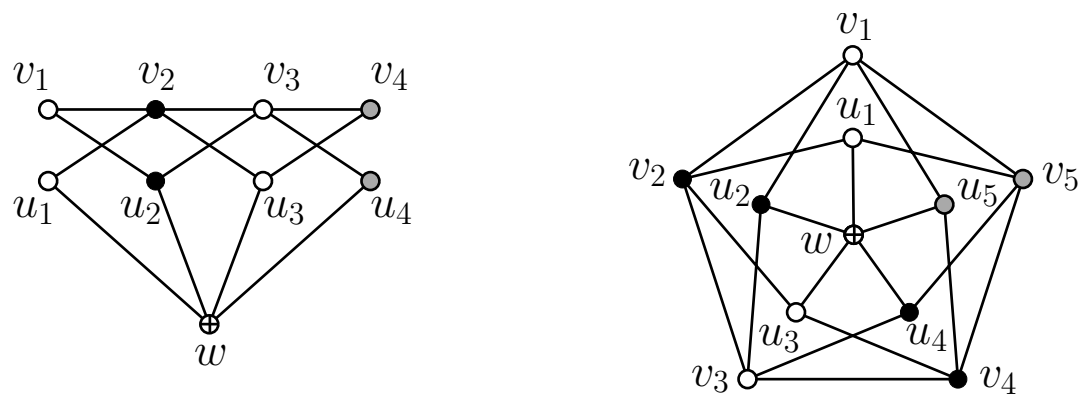

Figure 4: A pair of Mycieslki graphs. Left: $\mu\left(P_{5}\right)$. Right: $\mu\left(C_{5}\right)$.

Proof. It is sufficient to prove that every $k$-NL-coloring $\Pi=\left\{S_{1}, \ldots, S_{k}\right\}$ of $G$ can be extended to a $(k+1)$-NL-coloring of $\mu(G)$. Indeed, let $\Pi^{\prime}=\left\{S_{1}^{\prime}, \ldots, S_{k}^{\prime}, S_{k+1}^{\prime}\right\}$, where $S_{h}^{\prime}=S_{h} \cup\left\{u_{i}\right.$ : $\left.v_{i} \in S_{h}\right\}$, if $1 \leq h \leq k$, and $S_{k+1}^{\prime}=\{w\}$. By definition of $\mu(G)$, the sets $S_{i}^{\prime}$ are independent in $\mu(G)$, for every $i \in\{1, \ldots, k\}$. Besides, the set of colors of the neighborhood of $v_{i}$ in $\mu(G)$ is the same as for $v_{i}$ in $G$; and the set of colors of the neighbors of $u_{i}$ in $\mu(G)$ is the same as for $v_{i}$ in $G$ together with color $k+1$. Hence, vertices of $\mu(G)$ with the same color $i \in\{1, \ldots, k\}$ in $\mu(G)$, have neighborhoods with different sets of colors. From here, the inequality follows.

We show next that this bound is tight.

Proposition 8. If $G$ is a complete multipartite graph, then $\chi_{N L}(\mu(G))=\chi_{N L}(G)+1$.

Proof. By Theorem 3, we know that $G$ is a complete multipartite graph of order $n$ if and only $\chi_{N L}(G)=n$. In order to derive a contradiction, assume that there exists a $N L$-coloring $\Pi=\left\{S_{1}, \ldots, S_{n}\right\}$ of $\mu(G)$ using $n$ colors. Without loss of generality, assume that $w \in S_{1}$. Thus, vertices $u_{1}, \ldots, u_{n}$ are colored using at most $n-1$ colors, so that there are 2 vertices with the same color, say $u_{1}, u_{2} \in S_{2}$. Hence, vertices $v_{1}$ and $v_{2}$ must be adjacent in $G$, otherwise $u_{1}$ and $u_{2}$ would be false twins in $\mu(G)$, which is a contradiction, because both vertices have the same color in $\mu(G)$. Hence, $\left\{v_{1}, \ldots, v_{n}\right\} \subseteq N\left(u_{1}\right) \cup N\left(u_{2}\right)$, which implies that no vertex in $\left\{v_{1}, \ldots, v_{n}\right\}$ has color 2 . Thus, the $n$ vertices $v_{1}, \ldots, v_{n}$ are colored using at most $n-1$ colors in $\mu(G)$, which in turn implies that there exist two vertices, $v_{i_{1}}$ and $v_{i_{2}}$ with a same color. Thus, $v_{i_{1}}$ and $v_{i_{2}}$ must be non-adjacent in $G$, implying that $v_{i_{1}}$ and $v_{i_{2}}$ are false twins with the same color, which is again a contradiction. 


\section{Concluding remarks and open problems}

In this paper, we have introduced the neighbor-locating chromatic number of a graph, parameter that measures the minimum number of colors needed to paint a graph in such a way that any two vertices with the same color can be differentiated by the set of colors used by its neighbors. We believe that this new parameter will play a significant roll in the study of the structure of a graph, per se and by comparison with other previous known parameters such as the metric-locating chromatic number and the partition metric-location-domination number.

In view of the results obtained in the present paper and in the simultaneous work [1] (where we focus our attention on determining the neighbor-locating chromatic number of paths, cycles, fans, wheels and unicyclic graphs), we propose the following conjectures as future development directions in the study of neighbor-locating partitions of a graph.

The following conjecture arises from the results obtained in Sections 2 and 3.

Conjecture 1. For each pair $h, k$ of integers with $3 \leq h \leq k$, there exists a connected graph $G$ such that $\chi_{L}(G)=h$ and $\chi_{N L}(G)=k$.

In Section 5, we have addressed the behavior of the neighbor-locating chromatic number with respect to the join and the disjoint union of graphs. The following analysis is related to this behavior with respect to the Cartesian product $G \square H$ and the lexicographic product $G[H]$ of two arbitrary graphs $G$ and $H[22]$.

Given an NL-coloring of $G$ with colors $A=\left\{a_{1}, \ldots, a_{r}\right\}$ and an NL-coloring of $H$ with disjoint set of colors $B=\left\{b_{1}, \ldots, b_{s}\right\}$, consider the coloring of $G \square H$ using the set of colors $A \times B$ as follows: assign color $\left(a_{i}, b_{j}\right)$ to a vertex $(u, v)$ if $u$ has color $a_{i}$ in $G$ and $v$ has color $b_{j}$ in $H$. It is an easy exercise to prove that this is an NL-coloring of $G \square H$. Thus,

$$
\chi_{N L}(G \square H) \leq \chi_{N L}(G) \chi_{N L}(H) .
$$

In some way, this bound is best possible since the equality holds, for instance, when $G \cong$ $H \cong P_{2}$.

With regard to the lexicographic product, it is easy to check that

$$
\omega(G) \omega(H) \leq \chi_{N L}(G[H]) \leq \chi_{N L}(G) \chi_{N L}(H),
$$

where $\omega(G)$ denotes the clique number of $G$. However, we believe that, in general, these bounds are far from being tight. It is therefore an open problem to find tighter bounds. Another interesting problem is to determine the neighbor-locating chromatic number of both the Cartesian product and the lexicographic product of two graphs when one of them is a path or a complete graph or a cycle.

We propose the following conjecture involving both operations.

Conjecture 2. If $G$ and $H$ are connected graphs, then $\chi_{N L}(G[H]) \leq \chi_{N L}(G \square H)$.

In Section 6, we dealt with the problem of determining the neighbor-locating chromatic number of Mycielski graphs $\mu(G)$. We have shown that in general $\chi_{N L}(\mu(G)) \leq \chi_{N L}(G)+1$, but conjecture that the equality holds for any graph $G$.

Conjecture 3. For any graph $G, \chi_{N L}(\mu(G))=\chi_{N L}(G)+1$.

Proposition 8 supports this conjecture for the class of complete multipartite graphs. 


\section{References}

[1] L. Alcon, M. Gutierrez, C. Hernando, M. Mora, I. M. Pelayo: Neighbor-locatingchromatic number of pseudotrees. Preprint (2018).

[2] E. T. Baskoro And D. DarmajI: The partition dimension of corona product of two graphs. Far East J. Math. Sci., 66 (12) (2012), 181-196.

[3] A. Behtoei and M. Anbarloei The locating chromatic number of the join of graphs Bull. Iranian Math. Soc. 40 (6) (2014), 1491-1504.

[4] A. Behtoei and M. Anbarloei: A Bound for the Locating Chromatic Numbers of Trees. Trans. Comb., 4 (1) (2015), 31-41.

[5] A. Behtoei and B. Oмоомі: On the locating chromatic number of Kneser graphs. Discrete Appl. Math., 159 (18) (2011), 2214-2221.

[6] A. Behtoei and B. Omoomi: On the locating chromatic number of the Cartesian product of graphs. Ars Comb., 126 (2016), 221-235.

[7] J. Cáceres, C. Hernando, M. Mora, I. M. Pelayo, M. L. Puertas, C. Seara and D. R. Wood: On the metric dimension of Cartesian products of graphs. SIAM J. Discrete Math., 21 (2) (2007), 423-441.

[8] J. Cáceres, C. Hernando, M. Mora, I. M. Pelayo and M. L. Puertas: Locatingdominating codes: bounds and extremal cardinalities. Appl. Math. Comput., 220 (2013), 38-45.

[9] N. Campanelli and I. G. Yero: On some resolving partitions for the lexicographic product of two graphs. International Journal of Computer Mathematics, 94 (7) (2017), 1363-1374.

[10] G. G. Chappell, J. Gimbel and C. Hartman: Bounds on the metric and partition dimensions of a graph. Ars Combinatoria, 88 (2008), 349-366.

[11] G. Chartrand, D. Erwin, M. A. Henning, P. J. Slater and P. Zhang: The locatingchromatic number of a graph. Bull. Inst. Combin. Appl., 36 (2002), 89-101.

[12] G. Chartrand, D. Erwin, M. A. Henning, P. J. Slater and P. Zhang: Graphs of order $n$ with locating-chromatic number $n-1$. Discrete Math., 269 (2003), 65-79.

[13] G. Chartrand, L. Lesniak and P. Zhang: Graphs and Digraphs, (5th edition). CRC Press, Boca Raton, Florida, 2011.

[14] G. Chartrand, E. Salehi and P. Zhang: The partition dimension of a graph. Aequationes Mathematicae, 59 (2000), 45-54.

[15] D. Darmaji And R. Alfarisi: On the partition dimension of comb product of path and complete graph. AIP Conference Proceedings 1867 (2017), 020038.

[16] M. Fehr, S. Gosselin, and O. R. Oellermann: The partition dimension of Cayley digraphs. Aequationes Mathematicae, 71 (1-2) (2006), 1-18.

[17] H. Fernau, J. A. Rodríguez-Velázquez and I. GonzÁlez-Yero: On the partition dimension of unicyclic graphs. Bull. Math. Soc. Sci. Math. Roumanie, 57(105) (4) (2014), 381-391. 
[18] I. González Yero and J. A. Rodríguez-Velázquez: A note on the partition dimension of Cartesian product graphs. Applied Mathematics and Computation, 217 (7) (2010), 3571-3574.

[19] I. González Yero, M. Jakovac, D. Kuziak and A. Taranenko: The partition dimension of strong product graphs and Cartesian product graphs. Discrete Math., 331 (2014), 43-52.

[20] C. Grigorious, S. Stephen, R. Rajan and M. Miller: On the partition dimension of circulant graphs. Computer Journal 60 (2017), 180-184.

[21] C. Grigorious, S. Stephen, B. Rajan, M. Miller and A. William: On the partition dimension of a class of circulant graphs. Inform. Process. Lett. 114 (2014), 353-356.

[22] R. Hammark, W. Imrich and S. Klavar: Handbook of Product Graphs, (2nd edition). CRC Press, Boca Raton, Florida, 2011.

[23] F. Harary and R. Melter: On the metric dimension of a graph. Ars Combinatoria, 2 (1976), 191195.

[24] D. O. Haryeniand E. T. Baskoro: Partition Dimension of Some Classes of Homogenous Disconnected Graphs. Procedia Computer Science, 74 (2015), 73-78.

[25] M. A. Henning and O. R. Oellermann: Metric-locating-dominating sets in graphs. Ars Combin., 73 (2004), 129-141.

[26] C. Hernando, M. Mora, I. M. Pelayo, C. Seara and D. R. Wood: Extremal graph theory for metric dimension and diameter. Electron. J. Combin., 17 (2010), R30, 28pp.

[27] C. Hernando, M. Mora And I. M. Pelayo: Nordhaus-Gaddum bounds for locatingdomination. Eur. J. Combin., 36 (2014), 1-6.

[28] C. Hernando, M. Mora And I. M. Pelayo: Metric-locating-dominating partitions in graphs. Submitted. (https://arxiv.org/abs/1711.01086).

[29] I. Javaid, N. K. Raja, M. Salman and M. N. Azhar: The partition dimension of circulant graphs. World Applied Sciences Journal 18 (2012), 1705-1717.

[30] I. Javaid And S. Shokat: The Partition Dimension of Some Wheel Related Graphs. Journal of Prime Research in Mathematics 4 (2008), 154-164.

[31] E. C. M. Maritz And T. Vetrk: The partition dimension of circulant graphs. Quaestiones Mathematicae 41 (1) (2018), 49-63.

[32] J. Mycielski: Sur le coloriage des graphes. Colloq. Math. 3 (1955), 161-162.

[33] I. A. Purwasih, E. T. Baskoro, H. Assiyatun and D. Suprijanto: The Bounds on the Locating-Chromatic Number for a Subdivision of a Graph on One Edge. Procedia Computer Science, 74 (2015), 84-88.

[34] I. A. Purwasih, E. T. Baskoro, H. Assiyatun, D. Suprijanto and M. Baca: The locating-chromatic number for Halin graphs. Communications in Combinatorics and Optimization, 2 (1) (2017), 1-9. 
[35] J. A. Rodrguez-Velzquez, I. G. Yero, and D. Kuziak: The partition dimension of corona product graphs. Ars Combinatoria 127 (2016), 387-399.

[36] J. A. Rodríguez-Velázquez, I. González Yero and M. Lemanska: On the partition dimension of trees. Discrete Appl. Math., 166 (2014), 204-209.

[37] H. M. A. Siddiqui And M. ImRan: Computation of metric dimension and partition dimension of nanotubes. J. Comput. Theor. Nanosci., 12 (2015), 199-203.

[38] P. J. Slater: Leaves of trees. Proc. 6th Southeastern Conf. on Combinatorics, Graph Theory, and Computing, Congr. Numer., 14 (1975), 549-559.

[39] P. J. Slater: Dominating and reference sets in a graph. J. Math. Phys. Sci., 22 (4) (1988) 445-455.

[40] D. K. Syofyan,, E. T. Baskoro and H. Assiyatun: The Locating-Chromatic Number of Binary Trees. Procedia Computer Science, 74 (2015), 79-83.

[41] I. Tomescu And M. ImRAn: On metric and partition dimensions of some infinite regular graphs. Bull. Math. Soc. Sci. Math. Roumanie (N.S.), 52 (100) (4) (2009), 461-472.

[42] I. Tomescu, I. Javaid And I. Slamin: On the partition dimension and connected partition dimension of wheels. Ars Combinatoria, 84 (2007), 311318.

[43] D. Welyyanti, E. T. Baskoro, R. Simanjuntak and S. Uttunggadewa: On locatingchromatic number of complete n-ary tree. AKCE Int. J. Graphs Comb., 10 (3) (2013), 309-315.

[44] D. Welyyanti, E. T. Baskoro, R. Simanjuntak and S. Uttunggadewa: On Locatingchromatic Number for Graphs with Dominant Vertices. Procedia Computer Science,74 (2015), 89-92.

[45] D. Welyyanti, E. T. Baskoro, R. Simajuntak and S. Uttunggadewa: On the locatingchromatic number for graphs with two homogenous components. Journal of Physics: Conference Series, 893 (2017), 012040.

[46] I. G. Yero, M. Jakovac, D. Kuziak and A. Taranenko: The partition dimension of strong product graphs and Cartesian product graphs. Discrete Math. 331 (2014), 43-52.

[47] I. G. Yero and J. A. Rodrguez-Velzquez: A note on the partition dimension of Cartesian product graphs. Appl. Math. Comput. 217 (7) (2010), 35713574. 